\title{
Th2 cytokines increase kallikrein 7 expression and function in atopic dermatitis
}

\author{
Shin Morizane, MD, PhD¹, Kenshi Yamasaki, MD, PhD², Ai Kajita, MD¹, Kazuko Ikeda, MD¹, \\ Maosheng Zhan, MD' ${ }^{1}$, Yumi Aoyama, MD, PhD ${ }^{1}$, Richard L. Gallo, MD, PhD ${ }^{3}$, and Keiji \\ Iwatsuki, MD, PhD' \\ ${ }^{1}$ Department of Dermatology, Okayama University Graduate School of Medicine, Dentistry, and \\ Pharmaceutical Sciences, Okayama, Japan \\ ${ }^{2}$ Department of Dermatology, Tohoku University Graduate School of Medicine, Sendai, Miyagi, \\ Japan
}

${ }^{3}$ Division of Dermatology, Department of Medicine, University of California, San Diego and VA San Diego Healthcare System, San Diego, CA, USA

\section{Summary}

Here we report that Th2 cytokines increase kallikrein 7 expression and function in keratinocytes. Our finding describes a link between the Th2 environment and epidermal barrier dysfunction in atopic dermatitis.

\section{Keywords}

Th2 cytokines; tissue kallikrein 7; atopic dermatitis; serine protease

To the Editor;

Atopic dermatitis (AD) is a chronic pruritic inflammatory skin disorder characterized by a Th2 environment and epidermal barrier dysfunction. ${ }^{1,2}$ A primary defect in the epidermal barrier has been proposed as the initial event in the development of AD since loss-offunction mutations in filaggrin gene are associated with $\mathrm{AD} .{ }^{2}$ Excessive protease activity is another characteristic abnormality affecting the epidermal barrier in AD. Human tissue kallikreins (KLKs) are secreted serine proteases encoded by 15 genes located on chromosome 19, and in the skin, KLK5 and KLK7 are known as major serine proteases. These proteases degrade corneodesmosome proteins such as desmoglein 1, desmocollin 1, and corneodesmosin, leading to desquamation. ${ }^{3}$ KLK8 and KLK14 also have been shown to be involved in skin desquamation. ${ }^{3}$ While KLK5, KLK8 and KLK14, trypsin-like serine proteases, cleave carboxyl side of arginine or lysine, KLK7, a chymotrypsin-like serine protease, cleaves carboxyl side of tyrosine or phenylalanine. Increase in KLKs expression

(C) 2012 American Academy of Allergy, Asthma and Immunology. Published by Mosby, Inc. All rights reserved.

Address correspondence to, Shin Morizane, MD, PhD, Department of Dermatology, Okayama University Graduate School of Medicine, Dentistry, and Pharmaceutical Sciences. 2-5-1, Shikata-cho, Kita-ku, Okayama, 700-8558, Japan, Phone: +81-86-235-7282; Fax: +81-86-235-7283; zanemori@cc.okayama-u.ac.jp.

Publisher's Disclaimer: This is a PDF file of an unedited manuscript that has been accepted for publication. As a service to our customers we are providing this early version of the manuscript. The manuscript will undergo copyediting, typesetting, and review of the resulting proof before it is published in its final citable form. Please note that during the production process errors may be discovered which could affect the content, and all legal disclaimers that apply to the journal pertain.

Conflict of interest: The authors state no conflict of interest. 
has been reported in the epidermis of $\mathrm{AD}$, and the increase in KLK7 expression is prominent among KLKs. ${ }^{4}$ An association between genetic variations of KLK7 and AD have been also reported. ${ }^{3}$ Netherton syndrome patients who have a defect of lympho-epithelial Kazal-type related inhibitor (LEKTI), a serine protease inhibitor that could control excessive KLK activity, develop AD-like lesions. ${ }^{3}$ Correspondently, the overexpression of human KLK7 in murine epidermis results in chronic itchy dermatitis which is similar to chronic $\mathrm{AD}^{3}$ These findings suggest that an increase in KLK7 expression is involved in AD pathogenesis, though the regulation of KLKs expression in AD has not been fully studied.

We sought molecular mechanisms that might explain a KLK7 increase in AD and hypothesized that some inflammatory cytokines expressed in AD lesions induce KLK7 expression in keratinocytes. We stimulated normal human epidermal keratinocytes (NHEKs) with panels of inflammatory cytokines and revealed by ELISA analysis that Th2 cytokines; IL-4 (50 ng/ml) and IL-13 (50 ng/ml), significantly induced KLK7 expression in the cultured media but Th1 cytokines; IFN- $\gamma(100 \mathrm{U} / \mathrm{ml})$ and IL-12 $(50 \mathrm{ng} / \mathrm{ml})$, and Th17 cytokines; IL-17A (50 ng/ml), IL-22 (50 ng/ml), and IL-23 (50 ng/ml), did not (Fig 1A, see the Materials and Methods section in this article's Online Repository at www.jacionline.org). IL-33 (50 ng/ml), a pro-Th2 cytokine that has been recently highlighted as a strong inducer of Th2 cytokines in Th2 lymphocytes, ${ }^{1}$ did not affect KLK7 expression in NHEKs. We also confirmed that IL-4 (50 ng/ml) and IL-13 $(50 \mathrm{ng} / \mathrm{ml})$ significantly induced KLK7 mRNA expression in NHEKs (Fig 1B, see the Materials and Methods section in this article's Online Repository at www.jacionline.org). On the other hand, KLK5 and KLK8 expression was not altered by these Th2 cytokines (Fig 1C and D). KLK14 expression was not consistently detectable in cultured keratinocytes (data not shown). We also examined the effect on LEKTI, elafin, and secretory leukocyte protease inhibitor, which are serine protease inhibitors expressed by keratinocytes, but their expression was not affected (Fig 1E-G). Filaggrin and loricrin expression was significantly decreased by Th2 cytokines as previously reported (Fig $1 \mathrm{H}$ and I). ${ }^{5}$ Protease assays using specific substrates were performed to examine if serine protease activities increase in parallel to protein and mRNA level (see the Materials and Methods section in this article's Online Repository at www.jacionline.org). As expected, KLK7 activity, namely, chymotrypsin-like serine protease activity, was significantly enhanced by IL-4 $(50 \mathrm{ng} / \mathrm{ml})$ and IL-13 (50 ng/ml) (Fig 2A). Trypsin-like serine protease activity, the activity such as KLK5, KLK8, and KLK14, was not changed (Fig 2B). In addition to in vitro data, the analyses by real-time PCR and immunohistochemistry showed an increase in KLK7 expression in AD lesions compared with normal skin (Fig 2C and D, see the Materials and Methods section in this article's Online Repository at www.jacionline.org). Further, KLK7 protein level in the sera of AD patients significantly correlated with IL-4 amounts in the sera (Fig 2E, see the Materials and Methods section in this article's Online Repository at www.jacionline.org).

Th2 cytokines are generally expressed by Th2 lymphocytes, basophils, eosinophils and mast cells, and play roles in Th2 cell differentiation, IgE production, eosinophil recruitment, and so forth. ${ }^{1}$ These cytokines also affect epidermal barrier functions through STAT6 as IL-4 and IL-13 decrease expressions of filaggrin, loricrin, and involucrin in keratinocytes. ${ }^{5}$ Hatano et al. reported that IL-4 suppresses the expression of ceramide and cutaneous permeability barrier functions induced by TNF- $a$ and IFN- $\gamma$ and the recovery of cutaneous permeability barrier dysfunction in vivo. ${ }^{5,6}$ Desmoglein 3 expression is also inhibited by IL-4. ${ }^{5}$ IL-4 transgenic mice spontaneously develop AD-like dermatitis, which supports the importance of Th 2 cytokines in AD pathogenesis. ${ }^{1}$ We here report how Th2 cytokines could further alter the skin barrier by showing that IL-4 and IL-13 increase KLK7 expression and function in keratinocytes. Excessive protease activity is known to induce epidermal barrier 
dysfunction, thus the increase in KLK7 by IL-4 and IL-13 would count on the skin barrier disruption in $\mathrm{AD}$.

Interestingly, among skin KLKs, only KLK7 is a chymotrypsin-like serine protease, and others are trypsin-like serine proteases. Chymotrypsin-like serine protease KLK7 degrades human cathelicidin antimicrobial peptide LL-37, ${ }^{7}$ and decrease in LL-37 is documented in AD skin. ${ }^{8}$ Along with cathelicidin mRNA suppression by IL-4 and IL- $13,{ }^{9}$ the increase in KLK7 by IL-4 and IL-13 would further decrease antimicrobial skin barrier in AD. Taken together with previous reports suggesting the association between KLK7 and AD, the enhancement of protease activity through increased KLK7 expression by Th2 cytokines IL-4 and IL-13 might be an important factor for mechanical and chemical epidermal barrier dysfunction in AD.

\section{Acknowledgments}

Funding: Grant-in-Aid for Scientific Research (\#22791074)

\section{Abbreviations}
AD atopic dermatitis
KLKs kallikreins
LEKTI Lympho-epithelial Kazal-type-related inhibitor
NHEKs normal human epidermal keratinocytes

\section{References}

1. Brandt EB, Sivaprasad U. Th2 Cytokines and Atopic Dermatitis. J Clin Cell Immunol. 2011; 2

2. Cork MJ, Danby SG, Vasilopoulos Y, Hadgraft J, Lane ME, Moustafa M, et al. Epidermal barrier dysfunction in atopic dermatitis. J Invest Dermatol. 2009; 129:1892-1908. [PubMed: 19494826]

3. Ovaere P, Lippens S, Vandenabeele P, Declercq W. The emerging roles of serine protease cascades in the epidermis. Trends Biochem Sci. 2009; 34:453-463. [PubMed: 19726197]

4. Komatsu N, Saijoh K, Kuk C, Liu AC, Khan S, Shirasaki F, et al. Human tissue kallikrein expression in the stratum corneum and serum of atopic dermatitis patients. Exp Dermatol. 2007; 16:513-519. [PubMed: 17518992]

5. Ogg G. Role of T cells in the pathogenesis of atopic dermatitis. Clin Exp Allergy. 2009; 39:310316. [PubMed: 19040465]

6. Hatano Y, Terashi H, Arakawa S, Katagiri K. Interleukin-4 suppresses the enhancement of ceramide synthesis and cutaneous permeability barrier functions induced by tumor necrosis factor-alpha and interferon-gamma in human epidermis. J Invest Dermatol. 2005; 124:786-792. [PubMed: 15816837]

7. Yamasaki K, Schauber J, Coda A, Lin H, Dorschner RA, Schechter NM, et al. Kallikrein-mediated proteolysis regulates the antimicrobial effects of cathelicidins in skin. FASEB J. 2006; 20:20682080. [PubMed: 17012259]

8. Schauber J, Gallo RL. Antimicrobial peptides and the skin immune defense system. J Allergy Clin Immunol. 2009; 124:R13-R18. [PubMed: 19720207]

9. Howell MD, Gallo RL, Boguniewicz M, Jones JF, Wong C, Streib JE, et al. Cytokine milieu of atopic dermatitis skin subverts the innate immune response to vaccinia virus. Immunity. 2006; 24:341-348. [PubMed: 16546102] 

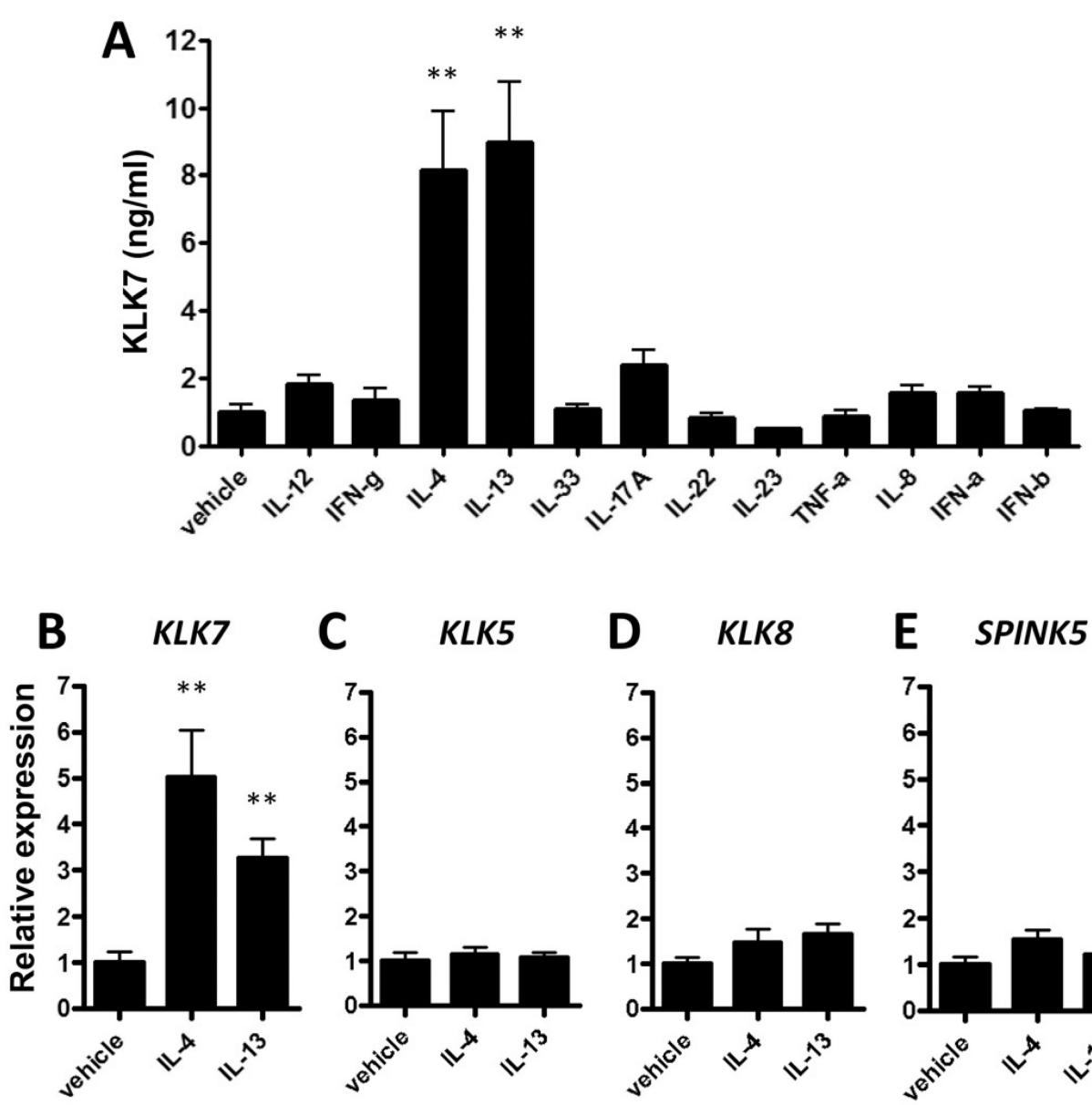

C KLK5

D $\quad K L K 8$

E SPINK5
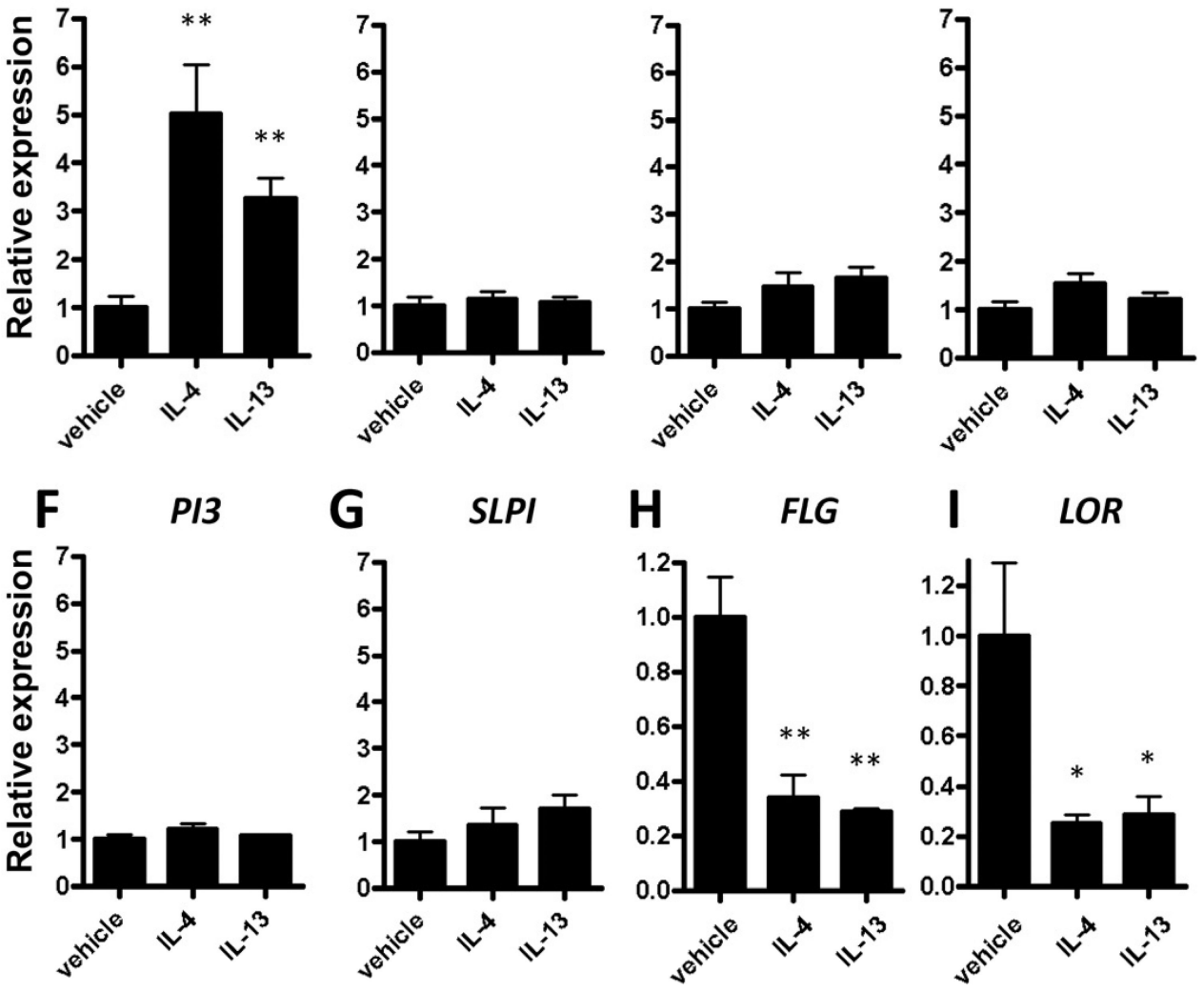

Figure 1.

(A) NHEKs were treated with inflammatory cytokines and KLK7 protein in the cultured media was measured by ELISA. (B-I) NHEKs were stimulated with IL-4 (50 ng/ml) or IL-13 $(50 \mathrm{ng} / \mathrm{ml})$ and the relative mRNA abundance of KLK7 (KLK7), KLK5 (KLK5), KLK8 (KLK8), LEKTI (SPINK5), elafin (PI3), SLPI (SLPI), filaggrin (FLG) and loricrin $(L O R)$ were analysed by real-time PCR. $* \mathrm{p}<0.05, * * \mathrm{p}<0.01$. Data are mean \pm SEM of triplicate samples and representative of three independent experiments. 
A
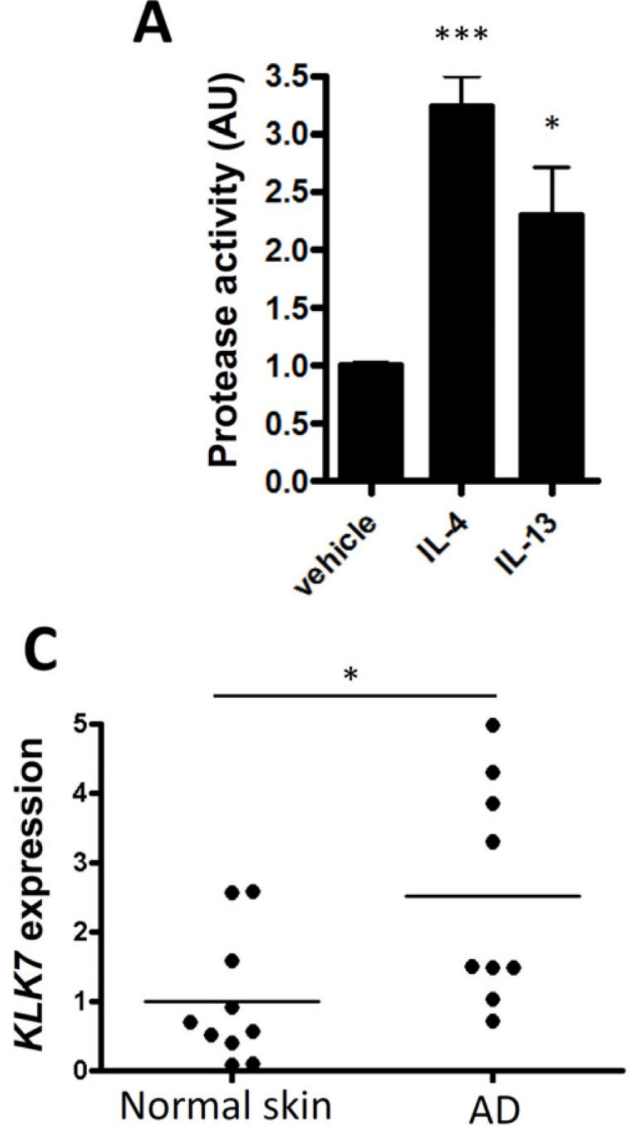

B

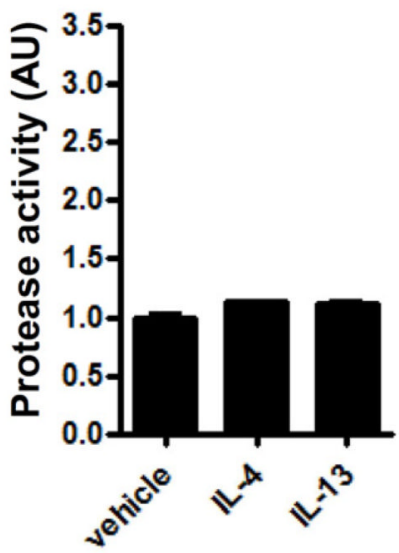

D

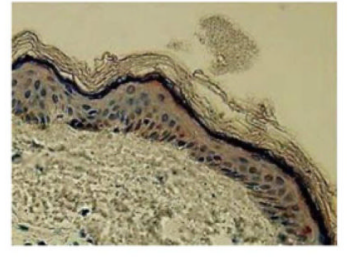

Normal skin

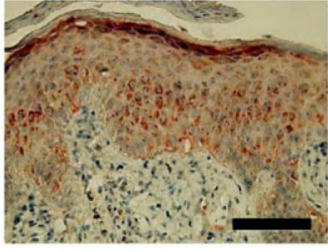

AD

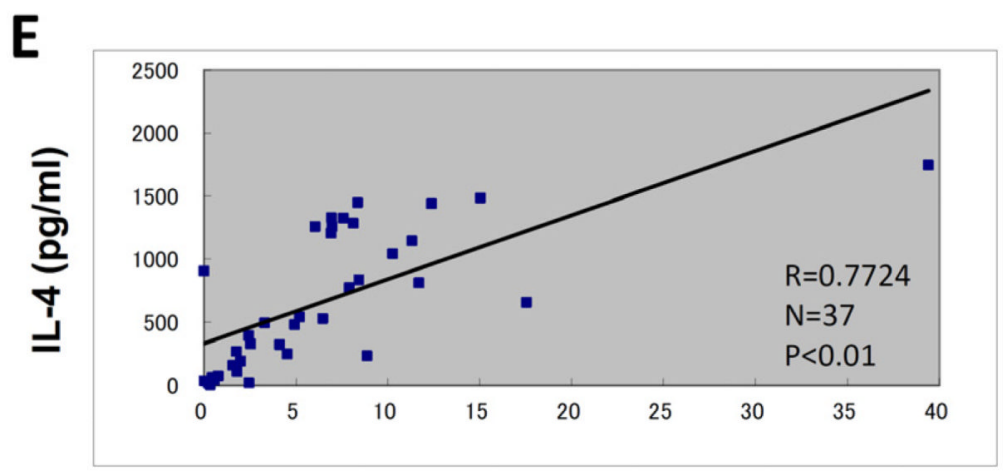

KLK7 (ng/ml)

Figure 2.

(A-B) NHEKs were stimulated with IL-4 (50 ng/ml) or IL-13 (50 ng/ml) and chymotrypsinlike (A) or trypsin-like (B) serine protease activity was measured using the specific substrates. $* \mathrm{p}<0.05, * * * \mathrm{p}<0.001$. Data are mean \pm SEM of triplicate samples and representative of three independent experiments. (C) KLK7 mRNA expression was analysed by real-time PCR and normalized with the mean of normal skins. *p<0.05. (D) KLK7 protein expression was examined by immunohistochemistry. Bar $=100 \mu \mathrm{m}$. (E) KLK7 and IL-4 level in the serum of AD patients were measured by ELISA and the correlation was analysed with Spearman's rank correlation coefficient. 